\title{
Soucis d'un officier colonial (Saint-Domingue, 1784-1785)
}

\section{G. Debien}

Volume 18, numéro 1, juin 1964

URI : https://id.erudit.org/iderudit/302345ar

DOI : https://doi.org/10.7202/302345ar

Aller au sommaire du numéro

Éditeur(s)

Institut d'histoire de l'Amérique française

ISSN

0035-2357 (imprimé)

1492-1383 (numérique)

Découvrir la revue

Citer ce document

Debien, G. (1964). Soucis d'un officier colonial (Saint-Domingue, 1784-1785).

Revue d'histoire de l'Amérique française, 18(1), 138-143.

https://doi.org/10.7202/302345ar d'utilisation que vous pouvez consulter en ligne.

https://apropos.erudit.org/fr/usagers/politique-dutilisation/ 


\title{
DOCUMENTS INÉDITS
}

\author{
I \\ SOUCIS D'UN OFFICIER COLONIAL \\ (Saint-Domingue - 1784-1785)
}

Les correspondances de familles venues des colonies sont sobres sur les événements publics, elles n'en parlent que dans les grandes circonstances et brièvement. Les questions d'argent, de succession, de partage, en forment le plus souvent le fond et les nouvelles des amis sont encore des affaires.

Cependant à Saint-Domingue - avec ses auberges surveillées, ses rapports de police, sa gazette, ses loges très tôt et nombreuses, sa société de pensée - c'est à peu près dans les seules lettres familiales qu'il faut chercher pour savoir les on-dit, tout ce qui se colportait, tout ce dont discutaient les gens à nouvelles, dans les grands repas, en voyage. L'histoire de l'opinion ne dispose pas de nombreuses sources.

Les deux lettres suivantes, de famille, présentent assez bien le genre de celles qui sont à la fois des lettres d'intérêt et l'écho des dires d'un moment; elles ont quelque chose, sur leur fin, d'une expression directe de ce dont on murmurait. De leur style, qui est très surveillé, pour mieux plaire, on ne dit pas qu'il ait la bride sur le cou.

De plus elles viennent d'un Canadien, ou plutôt d'un officier né au Canada, et il y est question de sa famille restée au Canada.

On ignore à qui exactement elles sont adressées.

Leur auteur est Pierre-Joseph Céloron de Blainville, capitaine au régiment du Cap; Pour les Canadiens ce nom n'est pas celui d'un inconnu, mais d'une famille militaire qui a servi longtemps en Nouvelle-France. 
Son père, Pierre-Joseph lui aussi, né à Montréal en 1693, était lui-même fils d'un capitaine des troupes du Canada, arrièrepetit-fils d'un secrétaire du roi. Il avait commandé au Détroit en 1743, à Niagara en 1744-1745; chargé par La Galissonnière en 1749 d'aller reprendre possession de la vallée de l'Ohio, il y avait fait construire les forts qui furent l'occasion de la guerre. Mort à Montréal en 1759, il avait laissé de ses deux mariages quinze enfants.

Notre correspondant, né le 1er juin 1747 à Montréal du second mariage - avec Catherine de la Pérelle - était entré au service à 12 ans. Légèrement blessé au Fort-Lévis en 1760 il y avait été fait prisonnier, puis conduit en Nouvelle-Angleterre, et de là en France où il fut réformé avec les troupes du Canada en 1761.

Il est sous-lieutenant dans la Légion de Saint-Domingue en 1767, à vingt ans, mais son avancement est particulièrement lent et terne: lieutenant en 1770 au même corps; en 1772 au régiment du Cap; capitaine en 1778; embarqué en juillet 1782 sur l'escadre du marquis de Vaudreuil il fait la campagne de la Nouvelle-Angleterre. Rentré au Cap en avril 1783, capitaine des chasseurs au régiment du Cap en octobre 1783; aide-majur de la place du Cap en 1785, major commandant de la place de Jérémie en 1787, chevalier de Saint-Louis en 1788, passé de la place de Fort-Dauphin en celle de Jérémie le 28 août 1788; lieutenantcolonel le 11 février 1792.

Revenu en France, infirme, il obtenait sa réforme avec une pension de retraite de 6000 francs "en considération de 52 ans 2 mois 10 jours de service y compris 26 ans 1 mois 5 jours dans la colonie de Saint-Domingue". Admis en qualité de chef de brigade aux Invalides le 5 frimaire an V (26 novembre 1797) il y mourut le 6 pluviose an XI (27 janvier 1803).

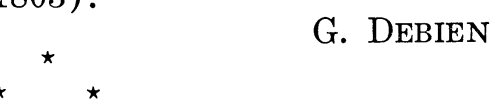

Du Cap français 28 octobre 1784

Mon aimable cousine

Je ne sais point augmenter mes torts en y joignant celui de les dissimuler ou de les affaiblir. Je me trouve aujourd'huy dans la position d'un pécheur qui joint à l'aveu de ses fautes le repentir le plus sincère de les avoir commises et qui n'a d'espoir que dans l'indulgence et la miséricorde divine. J'espère tout de la vôtre, et ce serait me punir trop rigoureusement que de vous refuser à 
oublier que depuis mon départ de France $^{1}$ je ne vous ai point écrit. Je vous le répète, mon aimable cousine, je n'userai d'aucune modification pour pallier une négligence aussi criminelle et au moment où je vous écrit mon cœur est en proie à l'idée la plus accablante d'être réduit à penser que vous jugerez que ce n'est qu'un motif d'intérêt qui me met la plûme à la main. Mais non, une femme qui réunit autant de qualités précieuses que vous ne croira pas que je sois parvenû à ce comble de dégradation, et j'ose me flatter que vous n'attribuerés mon éxactitude à venir qu'à un repentir bien sincère, et surtout à tous les sentimens que vous êtes faites pour inspirer. Celui qui se repent d'avoir mal fait est peut-être plus près du bien que celui qui ne s'en est jamais écarté. Voilà, ma trop aimable cousine, ma confession sans déguisement. Si vous la jugés digne de l'absolution j'acheverai mon confiteor, en me soummétant à la pénitence que vous m'imposerés; pourvû que ce ne soit pas la déffense de vous écrire de mon côté, et du vôtre de ne pas me répondre, car je vous avoue que mon cœur ne serait pas assez fort pour cela. J'ai reçu ces jours-cy une lettre du chevalier de Céloron, mon frère, ${ }^{2}$ qui a servi toute la guerre chés les américains, et que la paix a obligé de repasser en France pour y obtenir de l'emploi; il m'a fait part de ses succès dans ses démarches: il me marque qu'il est placé aidemajor de place à la Guadeloupe, ${ }^{3}$ et qu'il a été décoré de l'ordre de cincinnatus. J'ai lieu de croire aussi par sa lettre qu'il a été assés heureux pour vous rendre ses devoirs, quoiqu'il ne me le dise pas d'une manière positive. La nouvelle qu'il me donne de l'existance de madame Delaperrelle, ma grand mère ${ }^{4}$ et de $s a$ tendresse à retrancher sur ses besoins pour me prouver qu'elle m'a aimé audelà $d u$ tombeau dans lequel elle doit être ensevelie si j'en juge par la fin de sa lettre, a livré mon ame à tous les sentimens déchirans et doux de la confusion de la tendresse et $\mathrm{du}$ repentir. Puis-je avouer sans mourir de honte et de douleur que j'ai pû ne pas écrire à cette tendre et respectable ayeule depuis que je m'en suis séparé ? Puis je alléguer pour ma justification que depuis un séjour de 16 ans à Saint-Domingue, je n'ai

1 En 1767 ou 1768.

2 Paul-Louis Céloron de Blainville né le 2 mars 1753 à Détroit (FortPontchartrain) au Canada, sous-lieutenant dans le régiment de la Martinique en 1775, passé en Amérique en 1776 et admis comme volontaire, puis comme lieutenant dans le 1er régiment canadien continental, blessé à Saratoga. Capitaine en 1778, au siège de Savannah, prisonnier à Charleston; échangé seulement en novembre 1782 , retiré du service américain le 1er janvier 1783, capitaine aide-major à l'île Saint-Christophe en mars 1783.

3 Il venait d'être nommé aide-major de la Pointe-à-Pitre en février

1784. Il y resta jusqu'en décembre 1793 .

4 Grand'mère maternelle. 
reçu qu'une seule lettre de mon grand père, et que ce n'est que par mon oncle Laperrelle mort à la Martinique que j'ai appris que le premier était mort à Montléry, ${ }^{5}$ en m'apprenant que ma grand mère avait abandonné ce séjour sans me dire ou elle s'était retiré. A quelque tems dela j'apprends par mon frère aîné qui reste à Tours, que ma grand mère s'était retiré à Saint-Denys avec madame Villeray d'Artigny, et qu'elle y est morte. Il ajoute d'une manière douteuse quelle m'a laissé quelques choses et que vous avez eu la bonté d'ên être la dépositaire. Le chevalier de Céloron me le confirme par sa lettre datée de Tours du 24 avril 1784 et me dit avoir touché onze cents livres pour sa part et qu'il croit qu'il m'en revient deux milles pour la mienne. C'est un héritage plus précieux par la source d'où il me vient que par l'extreme besoin que j'en ai dans un pays ou les besoins de première nécessité ont toujours été excéssivement chers et surtout depuis la dernière guerre. Si le concours de mille circonstances me permettait d'aller en France je vous prierais, mon aimable cousine, de me continuer vos bontés et de me garder cet argent ou ces effets, car je ne sais en quoi cela consiste. Mais il m'est impossible dans ce moment, et peut-être même de quelques années d'entrevoir l'exécution d'un projet aussi séduisant que celui de revoir la France quoique ma santé en aye un grand besoin. J'ose donc vous prier, ma tendre cousine, de chercher sans vous donner cependant trop d'embarras, les moyens les plus prompts et les plus sûrs pour que je puisse toucher ici cet argent, et de joindre à tant de bontés de vôtre part celle de convertir en argent les effets qu'il pourrait y avoir. Je vais essayer de mon côté de vous faciliter en vous donnant quelques renseignements. J'ai un frère qui demeure à Saint-Rénan en Bretagne près de Brest ${ }^{6}$ qui était commissaire de la marine, qui est retiré qui je crois à l'honneur d'être connu de vous. Il connaît quelques négotiants du port de Nantes qui ont avec le Cap des relations continuelles d'affaires avec les commettans qu'ils ont dans cette ville. Je lui écris à ce sujet et le prie de vous instruire lorsqu'il se sera abouché avec quelqu'un de Nantes. Il est possible et cela est même sûr que ce négotiant ait un banquier à Paris ou autre correspondant que mon frere vous indiquerait lequel touchant la somme que vous lui remettriez, en la spécifiant argent de France ${ }^{7}$ tirerait une lettre d'échange sur le négotiant de Nante, et en dernier écrirait à son correspondant du Cap de

5 Où Céloron était resté entre la réforme des troupes du Canada (1761) et son départ pour la Légion de Saint-Domingue en 1767 ou 1768. tère).

6 Saint-Renan, chef lieu de canton de l'arrondissement de Brest (Finis-

$\tau$ Valant un tiers de plus que l'argent des Colonies. 
me compter en argent de la colonie la somme qu'il aurait touché en argent de France. Vous auriez la bonté de m'en instruire et si ce n'est pas trop exigé de vôtre complaisance je vous prierais de le faire par duplicata. C'est bien de la peine, ma chère cousine, mais que ne dois-je pas attendre de la bonté de vôtre cœur et de vôtre attachement innaltérable pour ma bonne maman. Voilà le seul moyen que je puisse vous fournir et ma manière de vous en faire part vous prouvera que je ne suis pas très habile dans les affaires et surtout celle d'argent. Si vous en avez de plus sûrs et surtout de moins pénibles pour vous, j'y souscris avec toute la confiance possible. Cette lettre vous sera peutêtre remise par le frère d'un de mes camarades, qui veut bien là lui adresser à Paris où il est à présent. Ce camarade est $\mathrm{Mr}$ De Vezien Desombrages, ${ }^{8}$ chevalier de Saint-Louis et capitaine au régiment, petit-neveu de madame de la Cossière. Nous logeons à côté l'un de l'autre et nous sommes très liés. C'est un garçon charmant qui unit à toutes les qualités qui font un bon officier celles qui constituent l'aimable homme. Je lui communiqué ma petite affaire; il me promet d'engager son frère qui loge chez madame de Chérutot, de vous remettre lui-même ma lettre, et peut-être vous procurera-t-il des moyens plus faciles pour l'objet qui m'intéresse. J'ai connu madame Desrosières, nièce aussi de madame de la Cossière et de $\mathrm{mr}$ le chevalier Devalière, un de nos généraux. ${ }^{9} \mathrm{Je}$ n'ai point été assez heureux pour lui faire une cour exacte parce qu'elle demeurait au Port-au-Prince et que ma résidence est au Cap. Je n'ai eu que l'avantage de passer quinze jours avec elle sur l'habitation de mr. le chevalier Delaval, colonel du régiment du Portau-Prince, dans un voyage que je fis dans cette partie de la colonie. Vous serez peut-être étonnée, mon aimable cousine, que depuis 16 ans que je suis dans cette colonie je n'aye pas songé à faire un mariage. Il peut y avoir de ma faute m'étant conduit comme tous les jeunes gens qui comptent trop sur les femmes des autres, néglige de se procurer un bonheur aussi doux. Mais d'un autre côté les ressources de cette colonie sont bien diminuées pour le militaire en général et surtout pour ceux qui n'ont pas l'avantage d'un grade supérieur. Les créoles riches, soit dit sans épigrame, sont en général sourdes au sentiment, mais très attentives aux hommages, et cette félicité douce qui n'a sa source que dans le cœur et qui se trouve dans

${ }^{8} \mathrm{Joseph}-\mathrm{François} \mathrm{Vézien,} \mathrm{chevalier,} \mathrm{sieur} \mathrm{des} \mathrm{Ombrages} \mathrm{(Saint-}$ Benoît-du-Sault, 1752 - Santiago de Cuba, 1803), avait fait lui aussi la campagne d'Amérique (Savannah).

9 Louis-Florent, chevalier de VALLIÈre, gouverneur de Saint-Domingue d'octobre 1772 à sa mort le 10 avril 1775 . 
le charme d'une société assortie, ne l'emporte pas dans leurs ames sur les distinctions de la vanité: daigner me rappeller au souvenir de mesdames de la Fontaine et leur faire agréer mes hommages respectueux. Un million de tendresses et d'amitiés à mes chers cousins. Recevez, ma tendre cousine, les vœux bien sincères que je fais pour vôtre bonheur et croyer que je n'y mets d'autres bonnes que celles de vôtre mérite et de mes sentimens réels pour vous, c'est vous prouver, je crois, toute l'étendue de mes souhaits.

Je suis avec respect

Madame et chère cousine

Votre très humble et très obéissant serviteur, Céloron.

mon adresse est à Mr. de Céloron, capitaine de chasseurs au régiment du Cap, au Cap français, isle de Saint-Domingue.

(à suivre) 